Meta

Journal des traducteurs

Translators' Journal

\title{
Index of Words and Topics in Volume 13
}

Volume 13, numéro 4, décembre 1968

URI : https://id.erudit.org/iderudit/003197ar

DOI : https://doi.org/10.7202/003197ar

Aller au sommaire du numéro

Éditeur(s)

Les Presses de l'Université de Montréal

ISSN

0026-0452 (imprimé)

1492-1421 (numérique)

Découvrir la revue

Citer ce document

(1968). Index of Words and Topics in Volume 13. Meta, 13(4), 221-224.

https://doi.org/10.7202/003197ar d'utilisation que vous pouvez consulter en ligne.

https://apropos.erudit.org/fr/usagers/politique-dutilisation/ 


\section{INDEX OF WORDS AND TOPICS IN VOLUME 13}

Absolute English, 2, p. 76.

Accuracy, 1, p. 7 .

Active core, 4, p. 197.

Actual, 3, p. 121

Adapter, 4, p. 191.

Addition, 1, p. 17.

Adjust (to), 2, p. 67.

Adjusting entry, 4, p. 193.

Adjustment, 1, p. 18, 20; 4, p. 193.

Aeronautics, 4, p. 196.

Affective meaning, 1, p. 15 .

Affix (to), 3, p. 130.

Agriculture, 1, p. 31.

Air foam stabilizer, 4, p. 192.

Alteration, 1, p. 17.

Ambulatory patient, 1, p. 18.

American slang, 1, p. $31 ; 3$, p. 144.

Americanisms, 3, p. 143

And / or, 3, p. 136.

Anglicised words and phrases, 3, p. 143.

Angry, 1, p. 14

Annoy, 1, p. 5.

Architecture, 1, p. 31.

Archive terminology, 3, p. 146.

Armature core, 4, p. 197.

Articulated seven-car consist, 4, p. 194.

Audience, 1, p. 8, 9 .

Author's intent, 1, p. 8.

Automatic stop, 2, p. 67.

Automation, 3, p. 146.

Automobile, 4, p. 196

Aviation, 1, p. 30 .

Back screen projection, 2, p. 68 .

Back-pack, 4, p. 192

Baffle, 3, p. 134

Baking powder biscuit, 3, p. 138.

Bar-mitzvah, 3, p. 119.

Behavioral meaning, 1, p. 17

Bibliography of translation, 4, p. 185.

Bi-level auto transporter, 2, p. 73.

Biological sciences, 2, p. 79.

Blow-valve, 2, p. 67

Blue, 1, p. 6

B'nai B'rith, 3, p. 120.

Bond, 4, p. 193 .

Borrowing, 1, p. 18

Bother, 1, p. 5.

Branch, 2, p. 67.

Breaking-in, 4, p. 192

Brick core, 4, p. 197.

Broadband exchange service, 2, p. 72.

Broadcast, 1, p. 22

Brown paper, 1, p. 12

Buckethandpump, 4, p. 192.

Budgetary control, 4, p. 193.

Building, 1, p. 31

Building industry, 4, p. 197.

Bull dozer, 2, p. 59 .

Bullhead rail, 2, p. 67

Cable core, 4, p. 197.

Call man, 4, p. 191

Campsite, 3, p. 135.

Canada Council, 4, p. 182.
Canadian literature, 4, p. 184.

Canadian-British words, 3, p. 143.

Canadianism, 2, p. 76.

Capitalized expenditures, 4, p. 194.

Casting, 3, p. 133.

Casual style, 1, p. 6 .

Charitable corporation, 3 , p. 135.

Chartered, 1, p. 25; 3, p. 139.

Chuck, 4, p. 190.

Cinema, 4, p. 196

Civil engineering, 1, p. 31 .

Class accent, 2, p. 48.

Cleaner, 3, p. 138

Clip, 2, p. 67

Closed end investment trust, 1, p. 27.

Closed-end fund, 1, p. 26.

Coffee cake, 3, p. 138.

Commercial, 3 , p. 147.

Commit (to) suicide, 1, p. 6.

Compound, 2, p. 59

Computer terms, 3, p. 146

Conceptual meaning, 1, p. 15.

Conference call, 2, p. 72 .

Confusing words, 2 , p. 76 .

Consultative marker, 1 , p. 6

Consultative style, 1, p. 6 .

Context, 1, p. 9 .

Control, 4, p. 194.

Convenient handling technique, 3, p. 130.

Copying, 1, p. 18.

Core, 4, p. 196

Corporation, 3, p. 135, 137.

Correcting entry, 4, p. 193.

Cosmetics, 2, p. 79.

Co-text, 1 , p. 9 .

Cottage cheese, 3, p. 137.

Counter clockwise, 2, p. 89.

Crate, 2, p. 68.

Crease resistant, 3, p. 138 .

Crocking, 3, p. 138 .

Cross-cultural research, 1, p. 2.

Cultural context, 1, p. 9.

Curtain board, 4, p. 192.

Data processing, 4, p. 197.

Data-phone set, 2, p. 72

Daydreaming, 1 , p. 5.

Deadman's handle, 2, p. 67.

Debenture, 4, p. 193.

Definition of translation, 4 , p. 185.

Déjà vu, 1, p. 17.

Delayed broadcast, 1, p. 23.

Delayed broadcasting, 1, p. 23 .

Delos, 4, p. 208

Depressed, 1, p. 6 .

Detergent, 2, p. 79.

Dictionary of National Biography, 4, p. 181.

Dirty, 1, p. 10.

Disbursement, 1, p. 27.

Disposable, 3, p. 128.

Dispose (to), 3, p. 129

Disarray (in), 1, p. 10.

Dolly-in, 2, p. 68.

Dolly-out, 2, p. 68 . 
Dome car, 4, p. 194.

Draft curtain, 4, p. 192.

Draft delinquent, 4, p. 195.

Draft evader, 4, p. 195.

Draft-dodger, 4, p. 195.

Drip dry, 3, p. 138.

Durable press, 3, p. 138.

Dynamic equivalence, 1, p. 7 .

Eating, 3, p. 147.

Ejaculatory impotence, 1, p. 12.

Elated, 1, p. 6.

Electrically operated valve, 2, p. 89.

Electronics, 2, p. 80; 3, p. 146; 4, p. 197.

Electrotechnology, 1, p. 31.

Elegance of form, 1, p. 8 .

Elimination period, 4, p. 201.

Ellipsis, 1, p. 6.

Engineering, 2, p. 56.

Environmental, 4, p. 200.

Equivalence, 1, p. 7.

Erective impotence, 1, p. 12.

Esquimo, 2, p. 79.

Esthetic aspect of language, 1, p. 8.

Etymo'ogy, 2, p. 76.

Evolution, 4, p. 191.

Explorers' journals, 4, p. 182.

External meaning, 1, p. 13.

Fac-simile, 2, p. 72.

Fading, 3, p. 134

Failed, 3, p. 141.

Faithfully yours, 3, p. 118 .

Familiarity with subject matter, 1, p. 12.

Feeder, 3, p. 134.

Fees for surveys, 3, p. 136.

Film library, 4, p. 189.

Financial terms, 3, p. 147.

Fire brigade, 4, p. 190.

Fire hydrant, 4, p. 190.

Fire line, 4, p. 192.

Fire plug. 4, p. 190

Fire prevention inspector, 4, p. 190.

Fire suppression organization, 4, p. 192.

First aid equipment, 4, p. 190.

First aid standpipe, 4, p. 190.

Fiscal, 3, p. 137.

Flush hydrants, 4, p. 190.

Foam compound, 4, p. 192

Foam liquid, 4, p. 192.

Foam stabilizing agent, 4, p. 192.

Foamed-in-place polyurethene, 4, p. 195

Foam-forming agent, 4, p. 192.

Foaming agent, 4, p. 192.

Foam-making compound, 4, p. 192.

Follow shot (lateral), 2, p. 68 .

Forcible entry, 4, p. 192.

Foreign language, 2, p. 47.

Formal equivalence, 1, p. 7

Formal marker, 1, p. 6.

Formal training, 1, p. 13.

French slang, 3, p. 144.

French-Canadian literature, 4, p. 180.

Frequency type telemeter, 2, p. 89.

Fringe benefits, 3, p. 137.

Full replacement, 1, p. 7.

Funded debt, 3, p. 136.

Fungi, 3, p. 138.

Gain measuring set, 2, p. 89.

Galosh, 1, p. 26.

Geographical names, 1, p. 31; 3, p. 146.
Geographical terms, 1, p. 31 .

German, 1, p. 31; 2, p. 77; 3, p. 146; 4, p. 203.

Governor General's Award, 4, p. 181.

Grants for translation, 4, p. 181.

Guilt, 1, p. 12

Hamburger, 3, p. 138.

Hand lamp, 2, p. 89.

Handling, 3, p. 130.

Hard core, 4, p. 197.

Hardware, 2, p. 73; 4, p. 198.

Health unit, 3, p. 135 .

Heat resistant, 3 , p. 138.

Heavy duty, 2, p. 68 .

Heraldry, 1, p. 30.

High, 1, p. 6 .

Holiday of Succoth, 3, p. 119.

Homophones, 3, p. 143.

Hook ladder, 4, p. 192.

Hose body, 4, p. 191.

Hose clamp, 4, p. 192

Hose line, 4, p. 191.

Hose station, 4, p. 190

Hoseman, 4, p. 191

Hot dog, 3, p. 138.

How often, 1, p. 12.

Hub, 3, p. 130 .

Hydrant key, 4, p. 192.

Hypodermic needle, 3, p. 128.

Ice cream soda, 3, p. 137.

Idiolect, 1, p. 6.

Idiosyncratic statistical feature, 1, p. 6.

Impotent, 1, p. 12.

Incendivity, 2, p. 56 .

Incentive rates, 2, p. 67 .

Indian pump, 4, p. 192.

Insight, 1, p. 18

Insurance, 4, p. 201.

Intentional meaning, 1, p. 16

Internal meaning, 1, p. 13 .

International French, 1, p. 11.

Ironing, 3, p. 138.

Jamaican English, 2, p. 76.

Judaism, 3, p. 118.

Keep away from heat, 3, p. 129.

Kiddush, 3, p. 121.

Kill (to) yourself, 1, p. 6.

Kinescope recording, 3, p. 133.

Korean, 3, p. 144.

Kosher, 3, p. 120.

Lack of translators, 4, p. 182.

Laminated steel core, 4, p. 196

Lateral follow shot, 2, p. 68 .

Law, 3, p. 146.

Lexical meaning, 1, p. 15 .

Light engine, 2 , p. 67.

Linguistic interference, 1, p. 13.

Linguistic meaning, 1, p. 14 .

Liquid level indicator, 2, p. 89.

Literary terms, 3 , p. 143 .

Live broadcasting, 1, p. 23.

Lobby, 3, v. 137.

Lobbying, 3, p. 137.

Lobbyist, 3, p. 137.

Local, 2, p. 68.

Local improvement, 3, p. 135.

Low-spirited, 1, p. 6.

Luer-lok, 3, p. 128.

Luganda, 2, p. 77.

Luggage travelling with the passenger, 2, p. 67. 
Machine translation, 2, p. 47.

Magnet core, 4, p. 196, 197.

Magnetic core, 4, p. 196, 197

Magnetized core, 4, p. 197.

Management, 2, p. 91 .

Managing, 2, p. 91.

Maranao, 2, p. 77

Marine, 4, p. 196.

Marker, 1, p. 6.

Market for translations, 4, p. 182.

Mask, 2, p. 69.

Matte, 2, p. 69

Meaning, 1, p. 13; 2, p. 47.

Mechanics, 4, p. 197.

Metal core, 4, p. 197

Metallurgy, 3, p. 146

Microwave, 2, p. 72

Microwave tower, 1 , p. 22

Milk shake, 3, p. 137.

Mining industry, 4, p. 197.

Mixing gear box, 4, p. 195

Mode, 1, p. 5.

Modern English, 2, p. 76.

Modifier, 1, p. 11 .

Modulating, 1, p. 18, 19.

Moisture proof, 3, p. 138

Moth proof, 3, p. 138

Music library, 4, p. 189.

Mutual open-end investment company, 1, p. 27.

Names, 1, p. 30

National Translation Center, 4, p. 208

Nautical terms, 3, p. 146.

Needle guard, 3, p. 130.

Newspaper library, 4, p. 189.

Nickname, 1, p. 30 .

No ironing, 3, p. 138

Non-pyrogenic, 3, p. 129

Non-reactive to tissue, 3 , p. 129

Non-toxic, 3, p. 129.

Nuclear core, 4, p. 197

Nuclear physics, 4, p. 197.

Nuclear tone, 2, p. 48

Nursery rhymes, 2, p. 50.

Nursing, 1, p. 24; 3, p. 139; 4, p 188.

Observation car, 4, p. 194.

Older Scottish tongue, 2, p. 76.

Open end investment trust, 1, p. $2 \%$.

Open-end fund, 1, p. 26.

Operational research, 2, p. 80; 3, p. 147.

Optical printer, 2, p. 69

Paired synonyms, 1, p. 6, 17.

Paper industry, 4, p. 196.

Fartial roplacement, 1, p. 7 .

Pass, 3, p. 133.

Passed, 3, p. 141

Peel apart (to), 3, p. 130

Peers and strangers, 1, p. 19.

Permanent fire fighter, 4, p. 191.

Permanent press, 3 , p. 138

Perma-pleat, 3 , p. 138

Personal terminology, 4, p. 201.

Petrol meter, 2, p. 67.

Phonotics, 2, p. 79.

Photography, 4, p. 196.

Phrasing, 2, p. 47 .

Pillar hydrant, 4, p. 190

Pit hydrant, 4, p. 190.

Plastic, 3, p. 146 .

Please, 1, p. 17
Plug door car, 2, p. 73.

Poetical terms, 2, p. 76.

Poetry, 4, p. 183.

Policy of translation, 4, p. 184

Post hydrant, 4, p. 190.

Potato chips, 3 , p. 138.

Power dome car, 4, p. 194.

Practical competence, 1, p. 13.

Prejudice, 3, p. 121.

Preparatory it, 3, p. 125.

Press, 3, p. 138.

Press (to), 3, p. 138.

Preview, 3, p. 132

Private meaning, 1, p. 15.

Processed cheese, 3, p. 138.

Projection, 3, p. 132.

Projector, 3, p. 132

Provincial English, 2, p. 76.

Psychiatric research instruments, 1, p. 4.

Public meaning, 1, p. 15

Pumper, 4, p. 191.

Pumping engine, 4 , p. 191.

Pumping equipment, 4, p. 190.

Pump-tank, 4, p. 192.

Punctuation, 2, p. 47 .

Quality in translation, 1, p. 7 .

Queer, 1, p. 5.

Quick motion, 2, p. 69

Quot:oons, 3, p. 145.

Radiator core, 4, p. 196.

Railway lexicon, 2, p. 67.

Railways, 4, p. 196.

Rank-bound, 1, p. 7

Rates for literary translation, 4, p. 182.

Realize (to), 3, p. 121.

Record library, 4, p. 189

Regional accent, 2, p. 48

Register, 1, p. 5 .

Relationship between translation and language learning, 2, p. 47

Remake, 3, p. 133

Rolled oats, 3 , p. 138

Remote broadcast, 1, p. 23.

Replacement, 1, p. 7, $18,20$.

Representation, 3, p. 132 .

Resistant to crocking, 3, p. 138.

Resistant to fungi, 3, p. 138 .

Resistant to light, 3, p. 138.

Resistant to perspiration, 3 , p. 138 .

Reverse lay, 4, p. 191

Reverse motion, 2, p. 69.

Rythm, 2, p. 48 .

Ring core, 4, p. 196

Roman Catholic Church, 3, p. 118.

Roof ladder, 4, p. 191.

Roof vent, 4, p. 192.

Rosin core solder, 4, p. 197.

Rubber overs, 1, p. 25.

Rubber overshoes, 1 , p. 25.

Rubber technology, 3, p. 146.

Rules, 1, p. 8

Salad dressing, 3, p. 138.

Salaries and wages, 3, p. 137.

Saltine crackers, 3, p. 138.

Sanforised, 3, p. 138.

Schedule, 3, p. 137.

Scholar, 3, p. 121.

Scientific instruments, 3, p. 146

Screening, 3, p. 132. 
Sense of humor, 1, p. 20 .

Shalom, 3, p. 120.

Shortening, 3, p. 138.

Shredded wheat, 3 , p. 138

S'il yous plaît, 1, p. 17

Similarity of response, 1, p. 8 .

Single throw, 2, p. 89.

Situational meaning, 1, p. 16.

Slang, 1, p. 30; 3, p. 143, 144.

Slash fire, 4, p. 192.

Slow motion, 2 , p. 69.

Small hose standpipe, 4, p. 190.

Snow-boot, 1, p. 26.

Snow-plow, 2, p. 68.

Software, 2, p. 73; 4, p. 198.

Sound, 4, p. 196.

Sound effects library, 4, p. 189.

Sound library, 4, p. 189 .

Source language, 1, p. 8 .

Spanish, 3, p. 144.

Spanner, 4, p. 192.

Special effects, 2, p. 69.

Spoken language, 2, p. 47.

Spoken mode, 1, p. 5 .

Spot remover, 3, p. 138.

Stability and riding comfort, 2, p. 72.

Stain resistant, 3, p. 138.

Stainless canula, 3 , p. 128

Starchless finish, 3, p. 138

Stationary orbit, 2, p. 72 .

Sterile, 3, p. 130.

Still library, 4, p. 189.

Straight lay, 4, p. 191

Strategic terminology, 1, p. 31

Stress, 2, p. 48.

Stress-point, 2, p. 50.

Style, 1, p. 6; 3, p. 143.

Substraction, 1, p. 17.

Sundae, 3, p. 137.

Superimpression, 2, p. 69

Superintendant, 2, p. 68.

Surnames, 1, p. 30.

Switcher, 2, p. 69.

Switching, 2, p. 69.

Switching panel, 2, p. 69 .

Synonyms, 3, p. 143 .

Syntactic meaning, 1, p. 15 .

Syringe, 3 , p. 130.

Tape library, 4, p. 189.

Target language, 1, p. 8

Techniques and procedures, 1, p. 13.

Telecommunications, 4, p. 197.

Telegraphese, 1, p. 6 .

Text, 1, p. 8

Textile, 3, p. 147

There, 3, p. 125.
Tired, 1, p. 12.

Toiletries, 2, p. 79.

Tonal features, 2 , p. 48.

Tones, 2, p. 48.

Tool room machinery, 3, p. 147.

Traffic manager, 2, p. 68.

Transistor, 3, p. 134.

Translating literally, 1, p. 18.

Translation, 2, p. 82

Translation (definition of), 1, p. 7 .

Translation and language learning, 1, p. 47.

Translator, 1, p. 8, 11 .

Translator courses, 1, p. 36.

Transmission, 1, p. 22.

Transmission line, 1, p. 22.

Transmission media, 1, p. 22.

Transmitter, 1, p. 22.

Transmitting antenna, 1, p. 22.

Transposing, 1, p. 18, 19.

TV drama, 4, p. 189

TV news, 4, p. 189.

Twist-push action, 3, p. 130.

Two-beat tone, 2, p. 48.

Typesetting, 3, p. 146

Tyre, 2, p. 67.

U-bolt, 2, p. 89

Unbounded translation, $1, \mathrm{p} . \%$

Underground hydrant, 4, p. 190.

Unit, 3, p. 128.

Unit of space, 2, p. 72

Unmagnetized core, 4, p. 197.

Up line, 2, p. 67.

Upper edge, 3, p. 130

Upset (to be), 1, p. 11 .

Usage and style, 3, p. 143

Valve core, 4, p. 196.

Variety of language, 1, p. 5.

Verbal meaning, 1, p. 17.

Vertical follow shot, 2, p. 68.

Vietnamese, 2, p. 77

Viewing, 3, p. 133

Volunteer fireman, 4, p. 191.

Vulgar tongue, 3, p. 143.

Wages, 3, p. 137

Wait (to) for an opening, 2, p. 67.

Waiting period, 4, p. 201.

Walkie-talkie, 2 , p. $89 ; 3$, p. 140

Wash and wear, 3, p. 138

Water proof, 3, p. 138.

Weird, 1, p. 5.

Welfare state, 3, p. 137

Well flat car, 2, p. 73.

Wishful thinking, 1 , p. 5 .

Woodworking terms, 2, p. 79.

Workmen's Compensation Board, 3, p. 135

Written language, 2, p. 47. 